\title{
The Energetics of Flux-Rope Prominence Models in Axially Symmetric Systems
}

\author{
J. Lin \\ Yunnan Observatory, Kunming, Yunnan 650011, China \\ T. G. Forbes and P. A. Isenberg \\ University of New Hampshire, Durham, NH 03824, USA \\ P. Démoulin \\ Observatoire de Paris, Meudon, France
}

\begin{abstract}
We analyze a prominence model containing a flux rope which is completely detached from the photosphere. The maximum magnetic energy which can be stored in the configuration does not exceed the openenergy limit proposed by Aly and Sturrock. However, a loss of ideal-MHD equilibrium can occur and create a current sheet which is likely to undergo rapid reconnection.
\end{abstract}

\section{Introduction}

According to Aly (1984) and Sturrock (1991), the energy stored in an axially symmetric system cannot exceed the energy of the fully opened magnetic field for any model where all the field lines are simply connected to the surface of the Sun. Mikić and Linker (1994) have confirmed that a simply connected arcade system with axial symmetry evolves towards the open configuration as the footpoints of arcade are sheared. Here we consider a prominence model which has some field lines that are not connected to the solar surface in order to see if it is possible to store energy in excess of the energy of the fully opened field. The model is based on an axially symmetric flux rope and a Sun-centered dipole.

\section{Evolution of the Model}

The model consists of two-dimensional equilibrium solutions in a spherical system with axial symmetry and with $r=R_{0}$ being the photospheric boundary. The coronal region $r \geq R_{0}$ is assumed to be perfectly conducting, and the gas pressure there is assumed to be negligible. The ring-like magnetic flux rope is suspended in the corona by a balance among tension, compression, and curvature forces. Evolution of the system takes place in two stages: The first stage stores energy, while the second releases it. During the storage phase, the evolution is slower than the reconnection, so current sheets cannot form due to dissipation. During the eruptive phase, mechanical equilibrium is lost and the system evolves rapidly over an Alfven time scale (i.e., $\leq 100$ s). The flux rope jumps to 
a higher altitude, releasing part of the magnetic energy stored in system, and a large-scale current sheet appears. This transition constitutes a catastrophe according to Thom (1972).

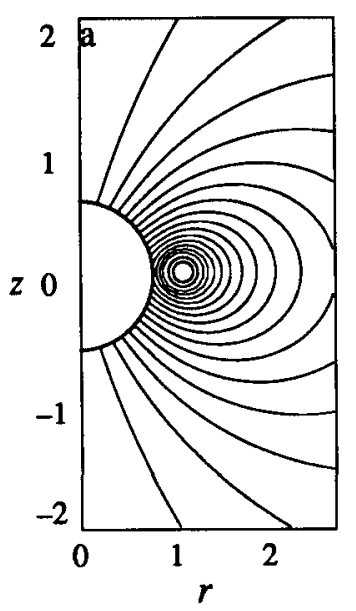

Figure 1. Magnetic field configuration. (a) prior to eruption, and (b) after eruption.

Initally, the flux rope is the only magnetic field source above the photospheric surface at $r=R_{0}$. For the model, we use the Lundquist solution to describe the current desity within the flux rope (Lundquist 1950 and Miller and Turner 1981), but outside the flux rope, the field is prescribed by the solution of Poisson's equation with the boundary condition:

$$
A_{\phi}\left(r=R_{0}, \theta\right)=\frac{m_{0} \sigma(t) \sin \theta}{R_{0}^{2}},
$$

on the photospheric surface. Here $m_{0}$ is a normalized constant, and $\sigma(t)$ is a function which slowly decreases with time. This boundary condition corresponds to a Sun-centered dipole of strength $m_{0} \sigma$ and, as $\sigma$ decreases, the configuration reaches a point where the system loses equilibrium. The flux rope jumps upwards, and a current sheet attached to the surface forms (Forbes and Isenberg 1991 and Isenberg et al. 1993). Figure 1 shows the configurations before and after the system loses equilibrium, while Figure 2 shows the equilibrium height of the flux rope $h$ as a function of $\sigma$ for an initial flux rope radius of $r_{0}^{o}=0.01 R_{0}$.

\section{Asymptotic Behavior and Energetics}

After the system develops a current sheet, we cannot determine a general analytic solution due to the additional mathematical complexity which is introduced, but we can investigate the asymptotic behavior of the system when the current sheet becomes very large and the flux rope is far from the Sun. In this limit, both $h$ and $q$, the height of the flux rope and the length of current sheet, 


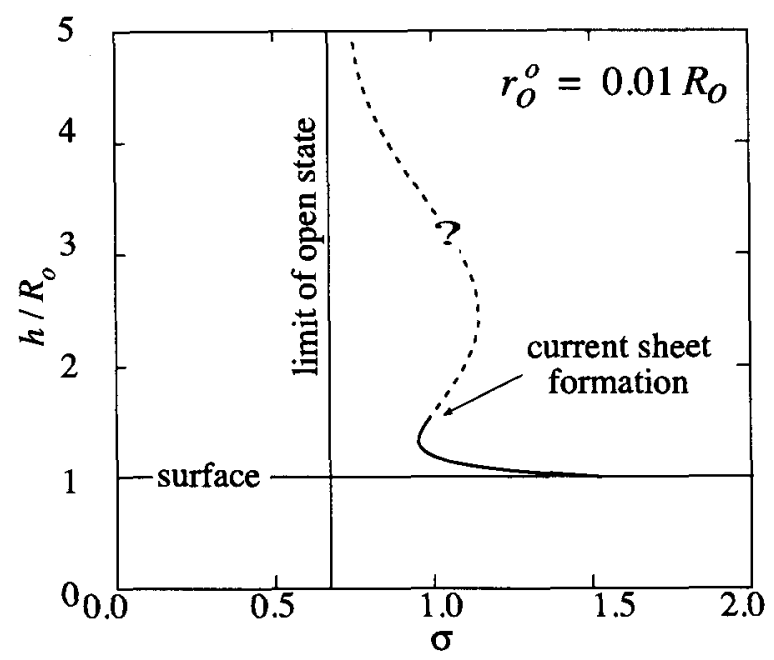

Figure 2. Equilibrium height $h$ as a function of dipole strength $\sigma$.

are much larger than $R_{0}$, and the Sun can be regarded as a point. This limit is in fact the open field limit, and it occurs at $\sigma=0.741$, as shown by the vertical line in Figure 2. Thus, the configuration becomes open if the attractive force supplied by Sun's dipole falls below a certain value. This behavior is similar to that found by Mikić and Linker (1994) for the opening of an arcade when its shear exceeds a particular value. Due to lack of a complete solution for the configuration with a current sheet of arbitrary size, the complete $h-\sigma$ relation remains unknown (dashed portion of the curve in Figure 2).

The magnetic energy stored in the system before losing equilibrium can be obtained in the way described by Isenberg et al. (1993). The result is shown in Figure 3 which plots the ratio $W_{\mathrm{t}} / W_{\mathrm{p}}$ against $r_{0}^{\circ} / R_{0}$, where $W_{\mathrm{t}}=W+W_{\mathrm{p}}$, $W_{\mathrm{t}}$ is the total magnetic energy, $W$ is the free magnetic energy, and $W_{\mathrm{p}}$ is the potential energy at the critical point. The ratio decreases as the flux-rope radius, $r_{0}^{\circ}$, declines, which is the opposite of the behavior of the 2-D models with translational symmetry. The change in behavior is caused by the curvature force (Shafranov 1966) which becomes infinite as $r_{0}$ tends to zero unless the current, and hence the energy $W$, also tend to zero at the same rate. The maximum energy ratio is 1.62 , which is just slightly smaller than 1.662 for the fully open field state.

\section{Conclusions}

We have analyzed a two-dimensional model for solar eruptions in a spherical system based on an axially symmetric flux rope and a Sun-centered dipole. The results show the system has catastrophic behavior when $r_{0}$ is sufficiently large. Catastrophe leads to sudden current sheet formation, but does not lead to a fully open field. The energy which can be stored in the system increases with $r_{0}^{\circ}$, contrary to what happens in models where the curvature force is absent. The 


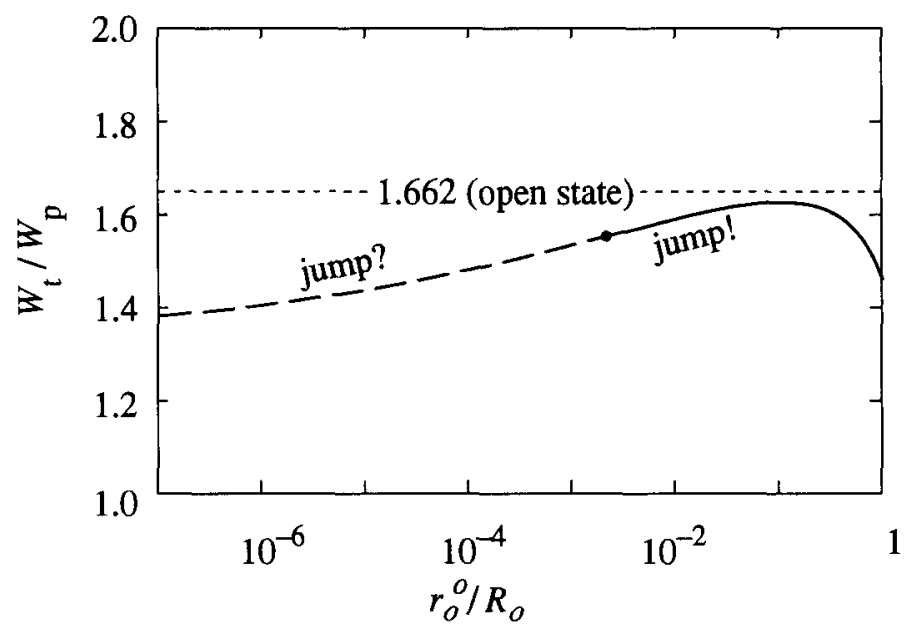

Figure 3. Magnetic energy $W$ of equilibria at the critical points as a function of the flux-rope radius $r_{0}^{o}$. Note that it is not clear if there is any catastrophe when $r_{0}^{\circ}$ is small (dashed curve), but catastrophe does occur for large $r_{0}^{o}$ (solid curve), and the energy stored prior to eruption can be very close to that stored in the fully open field.

maximum of $W_{\mathrm{t}} / W_{\mathrm{p}}$ is around 1.62 , which is smaller than 1.662 , the ratio for a fully open field. This result suggests that even for the models which have field lines that are not all connected to the surface, the theorem of Aly and Sturrock might still apply.

Acknowledgments. J. Lin gratefully acknowledges the support from K. C. Wong Education Foundation, Hong Kong. The work of T. G. Forbes was supported by NASA grants NAG5-4856 and NAG5-1479 to the University of New Hampshire, and NSF grant ATM-9596070 to Helio Research.

\section{References}

Aly, J. J. 1984, ApJ, 283, 349

Forbes, T. G. and Isenberg, P. A. 1991, ApJ, 373, 294 (Paper I)

Isenberg, P. A., Forbes, T. G. and Démoulin, P. 1993, ApJ, 417, 368

Lundquist, S. 1950, Ark. Fys., 2, 361

Mikić, Z. and Linker, J. A. 1994, ApJ, 430, 898

Miller, G. and Turner, L. 1981, Phys. Fluids, 24 (2), 363

Shafranov, V. D. 1966, Revs. of Plasma Phys., 2, 103

Sturrock, P. A. 1991, ApJ, 380, 655

Thom, R. 1972, Stabilité Structurelle et Morphogénése, Benjamin, New York 